\section{Local Differences in Skin Autofluorescence May Not Reflect Similar Differences in Oxidative Stress Exposure}

\section{To the Editor:}

Murray, et al report increased skin autofluorescence (AF) in patients with diffuse cutaneous systemic sclerosis (dcSSc) compared to persons with primary Raynaud's phenomenon and healthy controls, using measurements at 5 upper limb sites ${ }^{1}$. We agree that this might suggest a role for increased accumulation of advanced glycation endproducts (AGE), as markers of oxidative stress, in the skin of patients with dcSSc. However, their results are at odds with a lack of difference in skin AF we previously reported in patients with (diffuse and limited) cutaneous $\mathrm{SSc}^{2}$. Possible explanations for this discrepancy may be, first, that we used another device, the AGE reader, which has been specifically designed to assess skin AF, and second, we measured at only a single measurement site, recommended for this device, at the inner side of the lower arm below the elbow. The AGE reader has the advantage of being validated extensively in several conditions against dermal AGE levels in skin biopsies. To date, no such validation studies have been reported for the setup used by the authors. For the AGE reader, the lower arm site is preferred for reasons of limited epidermal thickness, usually lack of (fluorescent) hair and small traumata (scars are nonfluorescent for prolonged periods, possibly because of reduced AGE-related fluorescence of local newly formed collagen), and smaller (seasonal) changes in skin color (darker skin colors, despite the use of the ratio of excitation/emission, may affect skin AF). Figure 2 in Murray, et al, showing the differences in skin AF between groups, suggests that the differences are more obvious at the lower arm and wrist site than in the fingers and hand. This suggests that the measurement site may not explain the differences in results.

Further, in accord with the results of $\mathrm{Na}$, et $a l^{3}$ referenced by the authors, we also found in healthy controls higher skin AF levels at the inner ankle or calf healthy skin than at the inner arm. Thus it is questionable whether the differences in skin AF reported by Murray, et al for the different skin sites do reflect local differences in AGE accumulation and oxidative stress. This should be substantiated by validation studies against skin biopsy dermal AGE levels for different skin sites (and for different skin AF devices, depending on existing differences in optical setup).

Thus, caution is warranted in drawing conclusions on the observed differences in skin AF at different skin sites. To date, evidence is insufficient that local differences in skin AF reflect similar local differences in previous local exposure of the skin to oxidative stress.

MARTHA HETTEMA, MD, PhD, Rheumatologist, Medisch Spectrum Twente, Enschede; ANDRIES J. SMIT, MD, PhD, Vascular Medicine Internist, University Medical Center Groningen, Groningen, The Netherlands. Address correspondence to Dr. A.J. Smit, Department of Medicine, U3.129, University Medical Center Groningen, PO Box 30001, 9700 RB Groningen, The Netherlands. E-mail: a.j.smit@umcg.nl

\section{REFERENCES}

1. Murray AK, Moore TL, Manning JB, Griffiths CE, Herrick AL. Noninvasive measurement of skin autofluorescence is increased in patients with systemic sclerosis: An indicator of increased advanced glycation endproducts? J Rheumatol 2012;39:1654-8.

2. Hettema ME, Bootsma H, Graaff R, de Vries R, Kallenberg CG, Smit AJ. Skin autofluorescence, as marker of accumulation of advanced glycation endproducts and of cumulative metabolic stress, is not increased in patients with systemic sclerosis. Int J Rheumatol 2011;2011:417813.

3. Na R, Stender IM, Ma L, Wulf HC. Autofluorescence spectrum of skin: Component bands and body site variations. Skin Res Technol 2000;6:112-7.

J Rheumatol 2013;40:2; doi:10.3899/jrheum.121060 